\title{
Das Programm der Deutschen Gesellschaft für Gynäkologie und Geburtshilfe als Grundlage einer Qualitätssicherung in der operativen Gynäkologie
}

Gesundheitspolitische Maßnahmen und Reformen sind in der heutigen Zeit nicht mehr ohne Qualitätssicherung als integraler Bestandteil denkbar. Wie ein roter Faden durchzieht dieses Thema alle Bereiche des Gesundheitswesens, besondere aber den engeren medizinischen und den ärztlichen Bereich. Bei einer Definition einzelner ärztlicher Leistungen wie sie im Rahmen einer Gebührenordnung geschieht, ist die Notwendigkeit einer Beurteilung der Auswahl und Angemessenheit naheliegend.

Die Aufgabe der wissenschaftlichen Fachgesellschaften ist die Erforschung und Lehre der Medizin. Somit ist es auch ihre Pflicht, Kriterien für eine Beurteilung zu formulieren, ob das, was gelehrt wird, auch getan wird und ob das, was getan wird, richtig ist. Nichts anderes ist das Programm der Deutschen Gesellschaft für Gynäkologie und Geburtshilfe (DGGG) zur Qualitätssicherung in der operativen Gynäkologie. Qualitätssicherung im engsten ärztlichen Bereich ist der Kern eines jeden sehr viel umfangreicheren Qualitätsmanagements.

Nach guten Erfahrungen mit Maßnahmen zur Qualitätssicherung in der Geburtshilfe, der auch heute noch so genannten Perinatalerhebung, welche die deutsche Geburtshilfe international in eine führende Position brachte, beschloss der Vorstand der DGGG bereits im Jahre 1981 in Zusammenarbeit mit dem Institut für Medizinische Informationsverarbeitung (H.-K. Selbmann), Tübingen, ein ähnliches Programm auch für die operative Gynäkologie zu entwickeln.

In operativen Fächern fußen Programme zur Qualitätssicherung in aller Regel auf der Erfassung der Komplikationshäufigkeit bei einzelnen für das Fach typischen Operationen. In der Chirurgie erfolgt Qualitätssicherung beispielsweise mithilfe der Komplikationsrate bei nur wenigen sog. Traceroperationen. Auch in der operativen Gynäkologie folgten frühere Bemühungen diesem Prinzip [9]. Die Aussagekraft eines solchen Indikators erwies sich jedoch in der Gynäkologie als unzureichend, denn signifikante Abweichungen ließen sich nicht sicher erkennen. Die Erklärung hierfür liegt in einer primär niedrigen Komplikationsrate gynäkologischer Operationen und der in diesem Fach großen Zahl vielfältig kombinierter und damit nicht vergleichbarer Eingriffe. Wirklich vergleichbare Gruppen werden zu klein und sind statistisch nicht auswertbar.

Als aussagekräftiger Indikator erwies sich der Projektgruppe der DGGG $^{1}$ die Beurteilung der Indikationsstellung zur jeweils gewählten Operation. Nicht nur eine niedrige Komplikationsrate, sondern ebenso die Angemessenheit des Eingriffs im Verhältnis zum krankhaften Befund, also die richtige Indikationsstellung, sagen etwas über die Qualität ärztlichen Handelns aus. Als geeignet für diesen Qualitätsparameter erwiesen sich solche Operationen, die nicht mit anderen Eingriffen kombiniert sind, bei denen sich der krankhafte Befund postoperativ durch die histopathologische Untersuchung, also durch „harte“ Daten, präzise objektivieren lässt und bei denen der so gesicherte Befund die alleinige Indikation für die gewählte Operation war.

Es sei hier auf einen in der ärztlichen Diskussion, vor allem mit Pathologen, regelmäßig wiederkehrenden und oft hartnäckig verteidigten Irrtum, auf eine Fehlinterpretation des soeben dargelegten neuen Qualitätsindikators, hingewiesen. Es ist nicht die Tatsache der Befundsicherung durch eine histo-pathologische Untersuchung, so wichtig eine solche zweifellos ist, die den 
Qualitätsparameter ausmacht, sondern erst die Verknüpfung mit der Art des für diesen Befund ausgewählten operativen Eingriffs. Qualitätssicherung ist also nicht schon die Tatsache einer histopathologischen Untersuchung durch den Pathologen, sondern die richtige Indikationsstellung für die ärztliche Leistung und ihre Beurteilung nach den von der wissenschaftlichen Fachgesellschaft erarbeiteten Maßstäben.

Dieses bereits 1981/83 entwickelte Konzept wurde in einer ersten, von der Robert Bosch-Stiftung, Stuttgart, finanzierten Pilotstudie 1984/85 an zwanzig Kliniken jeder Größe erprobt [1]. Ausgewählt für die Bewertung der Aussagekraft des neuen Qualitätsindikators wurden die Eingriffe an der Cervix uteri, an den Adnexen und am Uterus unter Erfassung des gesamten übrigen operativen Spektrums der jeweiligen Kliniken, der Risiken, des Blutverlusts, der Verweildauer etc.

Das Konzept erwies sich als praktikabel und aussagekräftig, was an nur zwei Beispielen demonstriert sei. Eine Konisation wegen einer Ektopie der Portio als alleiniger Indikation ist, wenn überhaupt, in höchstens $2-5 \%$ akzeptabel. Es überraschte die Variationsbreite, die von Null bis $85 \%$ reichte. Eine ähnliche Variationsbreite fand sich bei manchen Adnexoperationen. Sie reichte seinerzeit bei der Extrauteringravidität von der laparoskopisch organerhaltenden Operation bis zur Exstirpation der gesamten Adnexe einschließlich des Ovars durch Laparotomie.

Nicht ausreichend beurteilen durch die histo-pathologische Diagnose ließ sich überraschenderweise die Indikationsstellung bei Eingriffen am Corpus uteri, beispielsweise wegen eines Myoms. Hier sagt die histo-pathologische Diagnose „Uterus myomatosus“ im Hinblick auf die zu beurteilende Indikationsstellung nicht viel aus. Ein kleines submukös gelegenes Knötchen kann durchaus eine Hysterektomie rechtfertigen, ein sehr viel größeres subserös gelegenes Myom aber nicht unbedingt. Auch die von manchen immer wieder geforderte Erfassung des Uterusgewichts kann hier aus diesem Grund nicht weiterhelfen.

Die Erkenntnisse der damaligen Pilotstudie wurden 1994 in einer sehr viel umfangreicheren, vom Bundesminister für Gesundheit finanziell geförderten Erhebung in 44 Kliniken weiter entwickelt und zur Anwendungsreife gebracht ${ }^{2}$ [2-5]. Es wurden wiederum das gesamte operative Spektrum der jeweiligen Klinik einschließlich der auch in der früheren Studie erfassten allgemeinen Daten, wie Komplikationsrate etc., erfasst und zusätzlich in drei Operationsgruppen, nämlich bei den Eingriffen an der Cervix uteri, an den Adnexen und an der Mamma u.a. mithilfe der histo-pathologischen Untersuchung die Indikationsstellung beurteilt. Es wurden 42 Qualitätsindikatoren formuliert und überprüft. 20 von diesen erwiesen sich als aussagekräftig und wurden zum „Programm der DGGG zur Qualitätssicherung in der operativen Gynäkologie“, jedoch erweitert um die nach $\S 301$ SGB V vorgeschriebenen Daten. Für die Anwendung in der Praxis

\footnotetext{
${ }^{1}$ Projektgruppe: L. Beck, Düsseldorf; H. Koester, Dortmund (Federführung); E. Koschade, Dachau; H.-K. Selbmann, Tübingen; S. Eichhorn (Deutsches Krankenhausinstitut) Düsseldorf.

2 Projektgruppe: D. Berg, Amberg; H. Koester, Dortmund (Federführung); R. Rauskolb, Northeim; P. Scheidel, Hamburg; H.-K. Selbmann mit M. Geraedts, Tübingen.
}

erwies es sich als vorteilhaft, das gesamte Programm auf einem einzigen Erhebungsbogen unterzubringen und eine brauchbare, inzwischen mehrfach überarbeitete, EDV-Version zu erstellen.

Das Programm der DGGG unterscheidet sich also von den Qualitätssicherungsprogrammen anderer Fachgesellschaften durch die komplette Erfassung sämtlicher Eingriffe und nicht nur vereinzelter Tracer-Operationen sowie vor allem durch die Beurteilung der Indikationsstellung zum Eingriff. Die Auswertung des sehr umfangreichen Datenmaterials von 42433 gynäkologischen Operationen ergab viele neue Erkenntnisse und auch Überraschungen, von denen hier einige beispielhaft geschildert seien.

Ein Drittel (33,1\%) aller Operationen waren Eingriffe an den Adnexen. In mehr als der Hälfte (53,1\%) war der Adnexbefund die alleinige Indikation für die Operation. In fast zwei Drittel (63,9\%) erfolgte eine Gewebeentnahme zur mikroskopischen Untersuchung. Diese wurden darauf hin ausgewertet, ob sich die Beurteilung der Indikationsstellung mithilfe der histo-pathologisch gesicherten Diagnose als Kriterium zur Qualitätssicherung eignet.

Neben anderen interessanten Aussagen überraschte es, dass in 23,0\% eine Follikel- oder Luteinzyste operiert wurde. Auch der Anteil unauffälliger Histologie war mit 27,9\% unerwartet hoch. Diese Daten sind jedoch zunächst nur ein Beispiel dafür, dass Zahlen allein bezüglich einer Qualitätssicherung gar nichts aussagen. Denn hierunter finden sich auch jene Adnexeingriffe, die in Zusammenhang mit einer anderen Operation, z.B. einer Hysterektomie, ausgeführt wurden. Bei diesen ist die Entfernung einer als Nebenbefund festgestellten Ovarialzyste aber durchaus sinnvoll.

Aufschlussreicher für die Beurteilung einer sorgfältigen Indikationsstellung sind jene Operationen, bei denen der Adnexeingriff der alleinige Eingriff war. Bei 3971 alleinigen Eingriffen an den Adnexen (ohne Tubensterilisierung) war der relative Anteil funktioneller Zysten als der dem Entschluss zur Operation zugrunde liegende Befund mit 29,5\% noch höher, fast $80 \%$ davon wurden laparoskopisch operiert. Weitere $14,9 \%$ waren histologisch unauffällig, zusammen also mit $44,4 \%$ fast die Hälfte aller alleinigen Adnexoperationen. Auch zeigte sich, dass es sich mit 78,1\% bzw. 67,6\% um typische laparoskopische Eingriffe handelt, die oft auch ambulant ausgeführt werden.

Diese Zahl wurde in anonymisierter Form den einzelnen Operationsstätten zugeordnet. Die Streubreite variierte bei einem Median von $31,3 \%$ von 6,3 bis $58,8 \%$. Als Referenzbereiche, innerhalb derer man positiv oder negativ auffällig wird, gelten bei Programmen zur Qualitätssicherung, wie z.B. auch bei der Perinatalerhebung, obere und untere Perzentilen des Medians. Für die operative Gynäkologie gilt als anzustrebender Referenzbereich der Wert der $25 \%$ besten Teilnehmer. Mit zunehmender Annäherung aller Teilnehmer an diesen Bereich steigt dann der Referenzbereich und die zu überspringende Messlatte wird immer höher. Je besser das Gesamtkollektiv wird, desto höher werden die Anforderungen. Bei den funktionellen bzw. unauffälligen Ovarialzysten war dieser so definierte Referenzbereich z.Zt. $<22 \%$. 
Die Abweichung dieser Rohdaten von den Referenzbereichen sagt zunächst jedoch nur, dass hier eine Auffälligkeit vorliegt, die mit dem bei der Perinatalerhebung seit langem bewährten Rückkopplungsverfahren durch die für den jeweiligen Ärztekammerbereich zuständige Fachgruppe der Projektgeschäftstelle abgeklärt werden muss. Würde man beispielsweise die Frequenz von Fällen mit assistierter Reproduktion mit dem Prozentsatz unauffälliger Histologie korrelieren, sollte letzterer entsprechend hoch sein und wäre ein Zeichen guter Qualität.

Die Ursachen für die bei funktionellen Zysten oft zu hohe Operationsfrequenz sind meist andere. Eine sonographisch unauffällige, einkammrige Zyste unter $10 \mathrm{~cm}$ Durchmesser ist zwar meist eine funktionelle Zyste, in 0,8\% aber auch ein Karzinom [7]. Sollte sich bei der nach sechs Wochen empfohlenen Kontrollsonographie, die den meisten Frauen wegen der dann in aller Regel erkennbaren Rückbildung der Zyste eine Operation ersparen würde, im Falle einer Operation ein Karzinom finden, wird dem Arzt trotz richtigen Handelns [8] wegen der für den Krankheitsverlauf unwesentlichen Verzögerung meist eine juristische Klage drohen. Auch die Erwartungshaltung des einweisenden Arztes mag unter Umständen von Einfluss auf die Entscheidung des Operateurs sein. Kann man es einem Operateur unter diesen Umständen verdenken, wenn er sich im Zweifel für eine großzügige Indikationsstellung zur Operation entscheidet?

Das Programm zur Qualitätssicherung schützt nicht nur die Patientin vor einer unnötigen Operation, sondern durch die ständige Rückkopplung mit der Fachgruppe der Projektgeschäftsstelle auch den Arzt vor juristischem Druck und ermöglicht ihm die fachlich richtige Entscheidung, unter sonographischer Kontrolle zunächst einmal abzuwarten. Der Anteil funktioneller Zysten lieBe sich hierdurch unter den anzustrebenden Anteil von unter $10-15 \%$ senken, jene Fälle nämlich, die wegen vorhandener Beschwerden, z. B. Stieldrehung, Blutung etc., eine Operation erforderlich machen. Als Nebeneffekt wäre die durch ein solches Vorgehen zu erreichende Kostendämpfung sinnvoller als jene durch z.B. eine Budgetierung.

Die Auswertung des umfangreichen Datenmaterials des Programms der DGGG ließ erkennen, dass vor allem in den Fällen, in denen die histo-pathologische Diagnose allein keinen sicheren Rückschluss auf eine angemessene Indikationsstellung, wie z. B. beim Uterus myomatosus, ermöglicht, das Lebensalter der Patientin ein aufschlussreiches Qualitätskriterium darstellt. Beim Dermoidkystom wird sich der Operateur bei der älteren Frau für eine Ovariektomie entscheiden, bei einer jüngeren Patientin aber möglichst ein organerhaltendes Vorgehen wählen. In der Erhebung der DGGG reichte die Variationsbreite organerhaltend operierter Dermoidkystome bei Frauen unter 40 Jahren bei einem Median von $80 \%$ von 0 bis $100 \%$.

Auch die vor allem in der Öffentlichkeit immer wieder geforderte Zurückhaltung bei der Hysterektomie, z.B. wegen eines Uterus myomatosus, ließ sich mit diesem Qualitätsindikator beurteilen. Die Streubreite einer Hysterektomie wegen einer gutartigen Erkrankung bei jungen Frauen unter 35 Jahren reichte bei einem Median von 4,3\% und einem Referenzbereich von $<3 \%$ von 0 bis $12,5 \%$.
Zahlreiche andere Qualitätsindikatoren ließen interessante Besonderheiten erkennen, z.B. die Anzahl der Blutübertragungen bei vergleichbaren Operationen. Große Abweichungen fanden sich u. a. bei der Frequenz der Hormonanalyse beim Mammakarzinom oder der Häufigkeit einer Röntgenlokalisation nicht tastbarer verdächtiger Befunde der Brust.

Diese wenigen Beispiele mögen genügen, um das Prinzip des Programms der DGGG zur Qualitätssicherung in der operativen Gynäkologie darzulegen. Viele der Abweichungen wurden den Teilnehmern erst bewusst, nachdem sie ihre eigene Position im Vergleich mit der Gesamtheit der anderen Teilnehmer und die Mediane und Referenzbereiche erfahren hatten. Der korrektive Effekt ähnelt dem der Perinatalerhebung.

Durch die Totalerfassung sämtlicher Operationen lassen sich die Schwerpunkte einer operativen Stätte und die Häufigkeit der dort praktizierten Operationen erkennen. Auch lassen sich unerwartete statistische Abweichungen feststellen und ggf. durch hierfür neu entwickelte Qualitätsindikatoren beurteilen und korrigieren. Das Programm ist also offen für eine ständige Fortentwicklung und Modifizierung.

Das Programm der DGGG wurde so entwickelt, dass es in gleicher Weise für stationäres und ambulantes Operieren benutzt werden kann. Der regelmäßig vorgetragene Einwand, der hierfür notwendige Zeitaufwand sei für ambulante Operationen zu groß, ist sachlich falsch. Durch die Hinzufügung eines einzigen Kästchens für die Code-Ziffer einer der vorgegebenen histo-pathologischen Diagnosen zum seit langem gebräuchlichen Bogen zur Qualitätssicherung beim ambulanten Operieren würde der gewünschte Effekt bereits bewirkt. Von Qualitätssicherung in der operativen Gynäkologie kann erst dann wirklich gesprochen werden, wenn sie für das gesamte Fach, also auch im ambulanten Bereich, in gleicher Weise angewandt wird.

Die Vorteile des Programms zur Qualitätssicherung in der operativen Gynäkologie für das ärztliche Handeln und auch bezüglich einer damit verbundenen Kostensenkung sollten eine umgehende flächendeckende Einführung vermuten lassen, doch nur in Hessen wurde es seit nunmehr über vier Jahren in etwa 90\% der Kliniken auf freiwilliger Basis eingeführt. In allen anderen Bundesländern wird die Anwendung in der von der DGGG erarbeiteten und erprobten Weise von mächtigen Interessengruppen, der GKV und der DKG, aus Gründen, die mit Qualitätssicherung wahrscheinlich wenig zu tun haben, blockiert. Die Forderung von Verbandsvertretern, die Thesen und Ergebnisse des mit der Studie von 1994 erarbeiteten Programms der DGGG müssten erst in einem Großversuch bestätigt werden, ist inzwischen bei weitem erfüllt. Die Ergebnisse des in Hessen, also einem ganzen Bundesland, eingeführten Programms der DGGG stimmen mit den Ergebnissen der Studie von 1994 vollkommen überein und bestätigen das Konzept [6]. Die auf Grund der derzeit noch gültigen Gesetzeslage vorgeschriebene Qualitätssicherung bei Fallpauschalen und Sonderentgelten in einigen Bundesländern unter der Regie der Servicestelle Qualitätssicherung (SQS) beim Deutschen Krankenhausinstitut praktizierte Form erzeugt zwar große Datenmengen, aber keine Qualität. 
Es ist zu wünschen, dass eine Qualitätssicherung, die diesen Namen verdient, in gleicher Weise für das ambulante und das stationäre Operieren im Rahmen einer Neuorganisation des Gesundheitswesens praktische Anwendung findet.

\section{Literatur}

${ }^{1}$ Eichhorn S, Koester H, Selbmann, HK. Qualitätssicherung in der operativen Gynäkologie. Ergebnisse der „Pilotstudie 1984“. Materialien und Berichte der Robert-Bosch-Stiftung, Bd.31. Gerlingen: Bleicher, 1989

2 Geraedts M, Koester H, Berg D, Rauskolb R, Scheidel P, Selbmann HK. Qualitätssicherung in der operativen Gynäkologie. Schriftenreihe des Bundesministeriums für Gesundheit, Bd.98. Baden-Baden: Nomos Verlagsgesellschaft, 1998
${ }^{3}$ Koester H. Qualitätssicherung in der operativen Gynäkologie. Arch Gynec Obstet 1985; 242: $37-41$

${ }^{4}$ Koester H. Qualitätssicherung in der operativen Gynäkologie aus klinischer Sicht. Zbl Gynäk 1995; 117: 670-673

${ }^{5}$ Koester H. Qualitätssicherung: Anspruch, Realität und Auswirkungen auf den medizinischen Alltag. Arzt u. Krankenhaus 1996; 69: $249-$ 256

${ }^{6}$ Koester H, Berg D, Rauskolb R. Qualitätssicherung in der operativen Gynäkologie und Perinatologie. Frauenarzt 1999; 40: 594-602

7 Osmers R, Kuhn W. Diagnostik und Therapie bei Ovarialtumoren. Frauenarzt 1995; 36: 925 -932

${ }^{8}$ Rehbock J, Kindermann G. Diagnostische Abklärung der klinisch oder sonographisch auffälligen Adnexe. Der Gynäkologe 1997; 30: 92 - 97

${ }^{9}$ Stark G. Qualitätssicherung in der operativen Gynäkologie. Arch Gynec Obstet 1985; 242: $37-41$ 